\title{
Aptidão física relacionada à saúde em adolescentes escolares
}

Devido à grande demanda de adolescentes inativos na população brasileira, dá-se a ideia de trabalhar com o tema aptidão física relacionada à saúde em adolescentes. $O$ trabalho objetivou analisar a aptidão física relacionada a saúde de adolescentes escolares da cidade de Porteiras/CE. Trata-se de um estudo de campo, descrito, com abordagem quantitativa e de corte transversal, realizado com escolares da cidade de Porteiras/CE. Com amostra de 98 escolares de ambos os sexos com idade de 15 e 16 anos. Utilizou-se a bateria de testes do PROESP-BR 2016. A análise estatística adotada foi a descritiva por distribuição de frequência através do SPSS versão 23 . O grupo se constituiu $40,8 \%$ de meninos e $59,2 \%$ de meninas, com média de idade $16,13 \pm 0,82$ anos. Com massa corporal média de $58,31 \mathrm{~kg}( \pm 9,25)$, estatura média $1,62 \mathrm{~m}( \pm 0,08)$, envergadura média de $1,64 \mathrm{~m}( \pm 0,10)$. Verificou-se que no índice de massa corporal $77,6 \%(H=30,7 \%$ e $M=46,9 \%)$ encontram-se nos padrões de zona saudável e $22,4 \%$ ( $H=10,2 \%$ e $M=12,2 \%)$ zona de risco. Na flexibilidade $89,8 \%$ ( $H=38,8 \%$ e $M=51 \%)$ na zona saudável e $10,2 \%$ $(\mathrm{H}=2 \%$ e $\mathrm{M}=8,2 \%)$ zona de risco. Na resistência abdominal $22,4 \%$ ( $\mathrm{H}=15,3 \%$ e $\mathrm{M}=7,1 \%$ ) estão em zona saudável e $77,6 \%$ ( $\mathrm{H}=25,5 \%$ e $\mathrm{M}=52 \%)$ zona de risco a saúde. Quanto a Resistência Cardiorrespiratória $13,3 \%$ ( $H=12,2 \%$ e $M=1 \%)$ encaixou-se nas zonas saudáveis e 86,7 ( $H=28,6 \%$ e $M=58,1 \%)$ em zonas de risco a saúde. Conclui-se que aptidão física relacionada a saúde da população estudada possui resultados preocupantes tanto na resistência abdominal como na capacidade cardiorrespiratória se localizando na zona de risco.

Palavras-chave: Atividade Física; Saúde; Adolescentes; Aptidão Física.

\section{Health-related physical fitness in school adolescents}

Due to the high demand for inactive adolescents in the Brazilian population, the idea of working with the topic of physical fitness related to health in adolescents is given. The work aimed to analyze the physical fitness related to health of schoolchildren in the city of Porteiras/CE. This is a field study, described, with a quantitative and cross-sectional approach, carried out with students from the city of Porteiras/CE. With a sample of 98 students of both sexes, aged 15 and 16 years. The PROESP-BR 2016 test battery was used. The statistical analysis adopted was descriptive by frequency distribution using SPSS version 23 . The group consisted of $40.8 \%$ boys and $59.2 \%$ girls, with mean age $16.13 \pm 0.82$ years. With average body mass of $58.31 \mathrm{~kg}( \pm 9.25)$, average height $1.62 \mathrm{~m}( \pm 0.08)$, average wingspan of $1.64 \mathrm{~m}( \pm 0.10)$. It was found that in the body mass index $77.6 \%(\mathrm{H}=30.7 \%$ and $\mathrm{M}=46.9 \%)$ are found in the healthy zone patterns and $22.4 \%(\mathrm{H}=$ $10.2 \%$ and $\mathrm{M}=12.2 \%)$ risk zone. In flexibility $89.8 \%(\mathrm{H}=38.8 \%$ and $\mathrm{M}=51 \%)$ in the healthy zone and $10.2 \%(\mathrm{H}=2 \%$ and $\mathrm{M}=8.2 \%)$ in the risk $z$ zone. In abdominal resistance $22.4 \%(H=15.3 \%$ and $M=7.1 \%)$ are in a healthy area and $77.6 \%(H=25.5 \%$ and $M=52 \%)$ health risk zone. As for Cardiorespiratory Resistance, $13.3 \%$ $(\mathrm{H}=12.2 \%$ and $\mathrm{M}=1 \%)$ fit in healthy areas and $86.7(\mathrm{H}=28.6 \%$ and $\mathrm{M}=58.1 \%)$ in risk areas the health. It is concluded that physical fitness related to the health of the studied population has worrying results both in abdominal resistance and in cardiorespiratory capacity located in the risk zone.

Keywords: Physical Activity; Health; Adolescents; Physical aptitude.

\section{Topic: Práticas Esportivas e de Lazer}

Reviewed anonymously in the process of blind peer.
Received: $14 / 03 / 2020$

Approved: 09/05/2020
Marcos Antonio Araujo Bezerra (iD)

Centro Universitário Dr. Leão Sampaio, Brasil

http://lattes.cnpq.br/4643352879633283

http://orcid.org/0000-0002-3385-4024

marcosantonio@leaosampaio.edu.br

Sandra Vieira do Nascimento

Centro Universitário Dr. Leão Sampaio, Brasil

http://lattes.cnpq.br/3725083774106607

sandrinha100@hotmail.com

Gabriela Gomes de Oliveira Bezerra

Centro Universitário Dr. Leão Sampaio, Brasil

http://lattes.cnpq.br/6330341118187369

gabriela.o.bezerra@gmail.com

\author{
Cícero Idelvan de Morais \\ Centro Universitário Dr. Leão Sampaio, Brasil \\ http://lattes.cnpq.br/9480395648727651 \\ idelvan@leaosampaio.edu.br \\ Cícero Rodrigo da Silva (iD) \\ Centro Universitário Dr. Leão Sampaio, Brasil \\ http://lattes.cnpq.br/8286964349591404 \\ http://orcid.org/0000-0003-4655-7982 \\ rodrigo@leaosampaio.edu.br \\ Cícero Cleber Brito Pereira (id \\ Centro Universitário Dr. Leão Sampaio, Brasil \\ http://lattes.cnpq.br/6554764959422181 \\ http://orcid.org/0000-0002-9402-9810 \\ cicerocleber@univs.edu.br
}

Referencing this:

BEZERRA, M. A. A.; NASCIMENTO, S. V.; BERREZA, G. G. O.; MORAIS, C. I.; SILVA, C. R.; PEREIRA, C. C. B.. Aptidão física relacionada à saúde em adolescentes escolares. Educationis, v.8, n.2, p.29-35, 2020. DOI: http://doi.org/10.6008/CBPC2318-3047.2020.002.0004 


\section{INTRODUÇÃO}

Devido à grande demanda de adolescentes inativos a ideia de trabalhar com a aptidão física relacionada à saúde em escolares surgiu através do público de adolescentes da população brasileira, observando o pouco interesse dos mesmos nas práticas de vidas saudáveis, tendo em vista que estes deveriam ser o público mais ativo, nota-se a falta de empenho pautada ao assunto.

Segundo Farias Junior et al. (2012) relata que a inatividade física é considerada um dos mais importantes problemas de saúde pública do século XXI. Além de representar um componente respeitável para um estilo de vida saudável e para promoção da saúde, a atividade física atua na prevenção de doenças crônicas não transmissíveis. A adolescência é um período crítico em relação à atividade física, pois a proporção de adolescentes fisicamente inativos é elevada, ainda que esse seja considerado o grupo populacional fisicamente mais ativo.

Com bases em dados do Ministério do Planejamento (IBGE, 2010) relata que o adolescente está tornando seu próprio lazer sedentário, sendo que estes, por sua vez, representam apenas uma parte do tempo total despendido pelos jovens em comportamentos sedentários, excluindo outras atividades sedentárias como o tempo sentado na escola e no deslocamento, por exemplo. Entretanto, apesar dessa delimitação, a Pesquisa Nacional de Saúde do Escolar mostrou que a prevalência de adolescentes expostos a pelo menos duas horas diárias de televisão é alta no Brasil (78,0\% no total, sendo $79,2 \%$ para o sexo feminino e $76,7 \%$ para o sexo masculino).

De acordo com Luciano et al. (2016) relata sobre a importância da pratica de atividade física realizada pela população adolescente também entrando na fase adulta a realização da atividade física regularmente, apresenta uma boa relação contrária com peso corporal e com doenças crônicas não transmissíveis além de gerar benefícios na aptidão física. A prática regular dessas atividades tem como objetivo principal auxiliar na promoção da saúde e melhora da qualidade de vida de crianças e adolescentes, além de ser primordial para o mantimento deste hábito de vida saudável estando em na idade adulta.

A importância de determinados padrões de distribuição da gordura corporal foi tratada por Lerario et al. (2002) relatando que ultimamente sabe-se que é a localização abdominal da gordura (obesidade central) que se tem uma demonstração mais adjunta a distúrbios metabólicos e risco cardiovascular. Conceitos regionais de obesidade, entre as quais a circunferência da cintura ou a razão entre as circunferências da cintura e do quadril (RCQ), são capazes de abastecer estimativa da gordura abdominal, que, por sua vez, está correlacionada à quantidade de tecido adiposo visceral.

Silva (2003), em meio às capacidades físicas voltadas à promoção da saúde, pode-se mencionar a força como uma das mais importantes, devido a sua semelhança com a redução de lesões, obtendo um acréscimo da autonomia de movimento, sendo, além disso, mostradas alguns enriquecimentos anatômicas e psicológicas. Santos et al. (2008), diz que força de resistência é a capacidade que o indivíduo possui de resistir a fadiga do organismo quando o trabalho de força é empregado em performances de longa duração.

Chaves et al. (2016) diz que a aptidão física relacionada à saúde conglomera parte aeróbico e não- 
aeróbico. Sendo que já permaneça constituído que a categoria aeróbica seja uma maravilhosa preditora de sobrevida em sujeitos de meia-idade, nos últimos 10 a 15 anos têm se congestionado destaques de que níveis altos de força muscular ou bons resultados no teste de sentar e levantar, um instrumento bem simples de avaliação dos componentes não-aeróbicos que observa o nível da aptidão física, sendo assim associados com um melhor prognóstico e, consequentemente, com uma menor mortalidade por todas as causas.

Assim, de acordo com Pereira et al. (2013) onde dizem que para uma redução da ampliação de doenças catalogadas ao sedentarismo se apresenta muito importante que esses indivíduos sejam orientados aos manifestos da cultura corporal do movimento de forma que se familiarizem com essas atividades, aprendendo a praticá-las e se envolver em um estilo ativo fisicamente tendo em vista que se levado pela vida toda é de suma importância.

A missão do profissional de educação, entre outras, é propor ações de intervenção social que promova o bem-estar físico e psicológico de indivíduos em uma determinada comunidade. Conhecer a realidade que se encontra um dado grupo facilita a descobrir os parâmetros que guiam o planejamento das ações necessárias. Este trabalho vem criar o cenário real em que se situam os adolescentes estudantes do ensino médio quanto ao perfil cineantropométrico classificando-os em níveis de aptidão física e composição corporal. O que possibilita deduzir sobre o nível de atividade física desse público. E assim sugerir a solução mais adequada a essa população. Assim, o objetivo da presente pesquisa foi identificar os parâmetros da aptidão física relacionadas a saúde de adolescentes escolares.

\section{MATERIAIS E MÉTODOS}

Para elaboração do estudo foram utilizadas informações associadas à aptidão física relacionada à saúde a partir de levantamento descritivo, de campo, de corte transversal, com abordagem quantitativa, de base populacional de escolares do município de Porteiras, Ceará, Brasil. A coleta dos dados foi realizada de 22 a 27 de março de 2019 e acompanharam as normas da Resolução 466/12 do conselho nacional de saúde. Após informados dos procedimentos, do objetivo do estudo, dos possíveis desconfortos, riscos e benefícios e questões éticas os sujeitos foram orientados a assinarem o Termo de Consentimento Livre Esclarecido (TCLE), garantindo-Ihes o anonimato sobre ouso das informações obtidas no estudo.

A população de referência para o estudo incluiu os escolares de ambos os sexos, entre 15 e 17 anos de idade, regularmente matriculados no ano letivo de 2019 na escola de ensino médio pertencente à rede de ensino público (estadual) do município de Porteiras, Ceará, Brasil. A Amostra foi composta por 98 escolares de ambos os sexos.

Utilizou-se como critério de inclusão a participação voluntária dos alunos, e foram adotados como critérios de exclusão: a) recusa em participar do estudo; b) não autorização dos pais ou responsáveis; c) ausência às aulas no dia agendado para a coleta dos dados; d) algum problema físico que o impedisse, temporária ou definitivamente, de realizar os testes motores.

Utilizou-se a bateria de testes do PROESP-BR 2016, proposto por Gaya et al. (2016), onde foram realizados os testes de aptidão física relacionada à saúde em adolescentes. Onde podem ser descritos como: 
massa corporal (peso), estatura (altura), envergadura, perímetro da cintura, índice de massa corporal (IMC), flexibilidade (teste sentar e alcançar), medida da razão cintura estatura (RCE), teste da corrida/caminhada dos 6 minutos e força/resistência abdominal (1 minuto). Os dados obtidos foram analisados através da estatística descritiva por distribuição de frequência, através do pacote SPSS na sua versão 23 para exploração completa dos dados (mínimo, máximo, media, desvio-padrão (DP) e percentual).

Os dados referentes aos resultados desta pesquisa abrangendo toda coleta de dados e análise, foram realizados no ano de 2019, tabulados separadamente e posteriormente foram criadas tabelas e gráficos, para uma melhor interpretação dos dados.

\section{RESULTADOS E DISCUSSÃO}

Atendidos os critérios de inclusão e exclusão do estudo, o grupo final de participantes foi constituído por 98 escolares (40,8\% de meninos e 59,2\% de meninas), com média de idade $16,13 \pm 0,82$ anos. A média da massa corporal dos pesquisados foi de $58,31 \mathrm{~kg}( \pm 9,25)$, enquanto a estatura apresentou uma média de $1,62 \mathrm{~m}( \pm 0,08)$. Em um estudo realizado por Contreira et al. (2016) mostra que as médias de massa corporal e estatura foram $43,50 \mathrm{~kg}( \pm 9,75)$ e $1,50 \mathrm{~cm}( \pm 0,08)$, respectivamente, assim notamos que os ambos os estudos se corroboram pelos resultados sendo analisada a mesma variável dos alunos estudados.

Em outro estudo feito por Godoi Filho (2006), o mesmo revelou que o sexo feminino apresenta estatura variável entre $119,9 \mathrm{~cm}$ e $155,0 \mathrm{~cm}$, por outro lado, os valores referentes encontrados para o sexo masculino variamente $121,0 \mathrm{~cm}$ a154,5cm sendo este um resultando que posteriormente se correlacionam aos níveis estudados. Outra variável estudada foi a envergadura que exibiu uma média de $1,64 m( \pm 0,10)$. Assim também observamos que em um estudo feito em escolares de 7 a 17 anos afirmam que valores de envergadura demonstram grande desvio padrão sem apresentar nenhuma diferença estatística (SANTOS et al., 2013).

Tabela 1: Análise descritiva para variáveis de AFRS em escolares de Porteiras/CE, 2019. (n=98).

\begin{tabular}{|l|l|l|l|l|}
\hline & Mín. & Máx. & $x$ & $D p$ \\
\hline Índice de Massa Corporal $\left(\mathrm{kg} / \mathrm{m}^{2}\right)$ & 16,18 & 29,88 & 22,07 & 3,04 \\
\hline Flexibilidade $(\mathrm{cm})$ & 17,0 & 64,0 & 38,21 & 10,98 \\
\hline Resistencia Abdominal (Rep) & 6,0 & 38,0 & 25,0 & 6,12 \\
\hline Aptidão Cardiorrespiratória (metros) & 433,0 & 1570,0 & 872,1 & 193,1 \\
\hline Relação Cintura Estatura - RCE & 0,3 & 0,7 & 0,439 & 0,05 \\
\hline
\end{tabular}

Legenda: Mín.= Mínimo - Máx.= Máximo $-x=$ média $-D p=$ Desvio Padrão.

Quanto a algumas variáveis presentes na pesquisa, na tabela acima representada por índice de massa

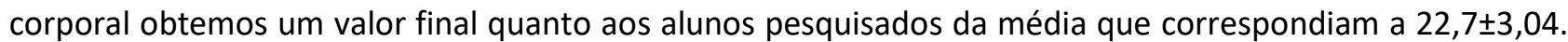
Já a flexibilidade exibiu um resultado onde a média foi de $38,21 \pm 10,98$. Onde a resistência abdominal mostrou valores como $25,0 \pm 6,12$. Sendo assim a aptidão cardiorrespiratória veio resultando em valores de, $872,1 \pm 193,1$. Por fim a relação cintura estatura evidenciou números como $0,439 \pm 0,05$.

De acordo com o estudo de Dumith et al. (2008), os valores médios encontrados para a amostra estudada em sua pesquisa no que se refere aos componentes de AFRS foram: IMC $=19,6 \mathrm{~kg} / \mathrm{m}^{2} \pm 3,6$; flexibilidade $=21 \mathrm{~cm} \pm 8 ; \mathrm{RML}=28 \pm 9$ repetições; capacidade aeróbia $=1.147 \pm 250$ metros. Contudo vemos que 
há uma grande pequena diferenciação quanto aos resultados encontrados na presente pesquisa, porém não notando muita diferença entre ambas, de acordo com os trabalhos estudados em relação as variáveis pesquisadas.

Já no estudo de Montoro et al. (2015) mostra que quanto a estas mesmas variáveis seus resultados

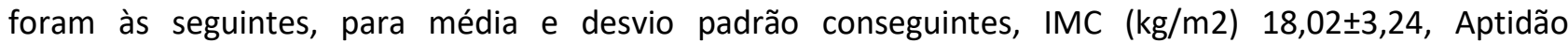

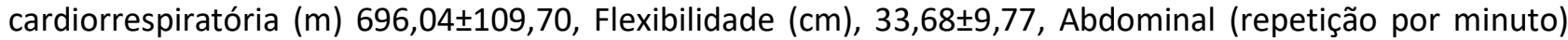
$18,01 \pm 6,4$. Observar-se que há alguns dados que se altera quanto ao presente estudos com alguns números que se elevam como a aptidão cardiorrespiratória e um pouco para menos o teste de resistência abdominal.

Tabela 2: Distribuição de frequência dos escolares nas avaliações da aptidão física relacionada à saúde quanto às zonas de saúde e risco. Porteiras, 2019.

\begin{tabular}{|c|c|c|c|c|c|}
\hline Avaliações & $\begin{array}{l}\text { Indivíduos Avaliados } \\
(\mathrm{n}=98)\end{array}$ & Classificação AFRS & $\begin{array}{c}\text { Total } \\
(n=98)\end{array}$ & $\begin{array}{c}\text { Meninos } \\
(n=40)\end{array}$ & $\begin{array}{c}\text { Meninas } \\
(n=58)\end{array}$ \\
\hline & & & $f(\%)$ & $f(\%)$ & $f(\%)$ \\
\hline \multirow[t]{2}{*}{ IMC } & \multirow[t]{2}{*}{$(n=98)$} & ZS & $77,6 \%$ & $30,7 \%$ & $46,9 \%$ \\
\hline & & ZR & $22,4 \%$ & $10,2 \%$ & $12,2 \%$ \\
\hline \multirow[t]{2}{*}{ Flexibilidade } & \multirow[t]{2}{*}{$(n=98)$} & ZS & $89,8 \%$ & $38,8 \%$ & $51 \%$ \\
\hline & & ZR & $10,2 \%$ & $2 \%$ & $8,2 \%$ \\
\hline \multirow[t]{2}{*}{ Abdominal } & \multirow[t]{2}{*}{$(n=98)$} & ZS & $22,4 \%$ & $15,3 \%$ & $7,1 \%$ \\
\hline & & ZR & $77,6 \%$ & $25,5 \%$ & $52 \%$ \\
\hline \multirow[t]{2}{*}{ Resistência Cardio } & \multirow[t]{2}{*}{$(n=98)$} & ZS & $13,3 \%$ & $12,2 \%$ & $1 \%$ \\
\hline & & ZR & $86,7 \%$ & $28,6 \%$ & $58,1 \%$ \\
\hline \multirow[t]{2}{*}{ RCE } & \multirow[t]{2}{*}{$(n=98)$} & ZS & $86,7 \%$ & $35,7 \%$ & $51 \%$ \\
\hline & & ZR & $13,3 \%$ & $5,1 \%$ & $8,2 \%$ \\
\hline
\end{tabular}

Legenda: $\mathrm{ZS}$ = zona saudável para AFRS; ZR = zona de risco para a saúde.

A tabela 2 traz consigo a classificação das variáveis quanto a aptidão física relacionada a saúde dentro das chamadas zonas de saúde e zonas de risco, nota-se que $77,6 \%$ dos escolares estudados (Mulheres=46,9\% e Homens $=30,7 \%$ ) se encaixam dentro de zonas saudáveis quanto a aptidão física relacionada a saúde. Nogueira et al. (2014) apresenta em seu estudo com população com as mesmas faixas etárias a esse estudo, resultados que evidenciam uma maior facilidade em atender os critérios de saúde para a variável de IMC.

Já Farias Júnior et al. (2003), apresentou em seu estudo uma prevalência de sobrepeso em adolescentes com idades entre 15 e 18 anos, em Florianópolis, de apenas $11,4 \%$, sendo mais elevada no sexo masculino $(14,8 \%)$ do que no sexo feminino $(8,0 \%)$. Neste componente, resultados positivos quando ao sexo feminino também foram encontrados em outras pesquisas (BURGOS et al., 2012; HENKES et al., 2013; MASCARENHAS et al., 2013).

Ainda de acordo com a tabela 2, no que se refere a variável flexibilidade, $89,9 \%$ dos escolares se encontram em zonas saudáveis (Mulheres=51\% e Homens=38,8\%). Em pesquisa desenvolvida por Glaner (2005), 40,8\% dos adolescentes não atingiram os critérios estabelecidos para a saúde na flexibilidade. Em estudos realizados com escolares de diferentes regiões do Brasil, foram encontrados valores médios superiores na flexibilidade para o sexo feminino em todas as idades analisadas (PELEGRINI et al., 2011).

Já sobre a resistência abdominal o estudo revelou resultados não favoráveis a saúde dos escolares, pois $77,6 \%$ da amostra (Mulheres=52\% e Homens=25,5\%) apresentaram valores dentro das zonas de risco a 
saúde da população estudada. Diferenças na força/resistência abdominal são observadas em todas as idades com melhor desempenho para meninos em relação às meninas, e durante o estirão da adolescência eles obtêm ganhos mais rápidos na força resistência abdominais devido ao aumento dos níveis de testosterona (MALINA et al., 2009).

Pelegrini et al. (2011) em seu estudo encontrou mais de 50\% dos escolares classificados dentro de zonas de risco, sendo os valores encontrados nesse estudo se equiparam aos dados encontrados pelo do autor. No que se trata da resistência cardiorrespiratória destaca-se que nesta variável foram encontrados os valores mais altos dentro de zonas de risco a saúde, onde se encaixaram $86,7 \%$ da amostra (Mulheres=58,1\% e Homens=28,6\%). Os dados são confirmados por outras pesquisas avaliando a população infantil e adolescente, as quais também encontraram resultados insatisfatórios para a condição cardiorrespiratória (HENKES et al., 2013).

A partir do quadro apresentado, observa-se um estado de alerta quanto às condições cardiorrespiratórias de crianças e adolescentes, uma vez que este componente da aptidão física tem maiores implicações para a saúde, pois se refere à capacidade das pessoas de sustentar a realização de atividades vigorosas (HAYWOOD et al., 2010).

Sobre os resultados encontrados para o novo teste do Projeto Esporte Brasil - PROESP em sua versão 2016 encontra-se o teste de Razão Cintura Estatura (RCE), onde 86,7\% da amostra (Mulheres=51\% e Homens=35,7\%) apresentaram níveis satisfatórios dentro de zonas saudáveis. As decorrências práticas do presente estudo podem ser examinadas no estímulo ao bem-estar no conjunto escolar, levando em consideração que neste ambiente é plausível desenvolver atuações e interferências precisas, tendo em vista potencializar a aptidão física relacionada à saúde dos escolares.

\section{CONCLUSÕES}

No presente estudo pode-se concluir que aptidão física relacionada a saúde da população estudada encontra-se com resultados preocupantes no que se trata das capacidades físicas de resistência abdominal podendo indicar predisposição à ocorrência de doenças de natureza hipocinética na vida adulta e da capacidade cardiorrespiratória, onde a sua grande maioria se localiza dentro de zonas de risco a saúde. Portanto estão sujeitos a muitas doenças futuras, tendo em vista que os mesmos poderiam ter evitando tal transtorno, fazendo o uso de fatores diários saudáveis sempre presente em seu dia a dia.

Dessa forma sugere-se que novas pesquisas sejam realizadas no sentido de abordar uma quantidade maior de sujeitos, inserirem variáveis como aspectos motivacionais para prática de exercícios físicos, verificarem a relação com os aspectos cognitivos e inserir variáveis clinicas para verificar o efeito do nível de aptidão física sobre aspectos hormonais.

\section{REFERÊNCIAS}

BURGOS, M. S.; REUTER, C. P.; TORNQUIST, L.; PICCIN, A. S.; RECKZIEGEL, M. B.; POHL, H. H.; BURGOS, L. T.. Perfil de aptidão física relacionada à saúde de crianças e adolescentes de 7 a 17 anos. J. Health Sci. Inst., v.30, n.2, p.171-175, 2012. 
CHAVES, T. O.; BALASSIANO, D. H.; ARAÚJO, C. G. S.. Influência do hábito de exercício na infância e adolescência na flexibilidade de adultos sedentários. Revista Brasileira de Medicina do Esporte, v.22, n.4, p.256-260, 2016.

CONTREIRA, A. R.; PIZZO, G. C.; ROCHA, F. F.; LAZIER-LEÃO, T. R.; CARUZZO, A. M.; COPETTI, F.; BELTRAME, T. S.. Perfil de Aptidão Física Relacionada à Saúde em Escolares. Saúde e Pesquisa, Maringá, v.9, n.2, p.309-315, 2016.

DUMITH, S. C.; AZEVEDO JUNIOR, M. R.; ROMBALDI, A. J.. Aptidão física relacionada à saúde de alunos do ensino fundamental do município de Rio Grande, RS, Brasil. Rev. Bras. Med. Esporte, Niterói, v.14, n.5, p.454-459, 2008

FARIAS JUNIOR, J. C.; LOPES, A. S.. Prevalência de sobrepeso em adolescentes. Rev. Bras. Ci. e Mov., Brasília, v.11, n.3, p.77-84, 2003.

FARIAS JUNIOR, J. C.; LOPESII, A. S.; MOTAIII, J.; HALLAL, P. C.. Prática de atividade física e fatores associados em adolescentes no Nordeste do Brasil. Rev. Saúde Pública, São Paulo, v.46, n.3, p.505-515, 2012.

GAYA, A.; GAYA, A. R.. Projeto esporte Brasil: manual de testes e avaliação. Porto Alegre: UFRGS, 2016.

GLANER, M. F.. Aptidão física relacionada à saúde de adolescentes rurais e urbanos em relação aos critérios de saúde. Rev. Bras. Educ. Fís. Esporte, v.19, n.1, p.13-24, 2005.

GODOI FILHO, J. R. M.. Índices antropométricos e níveis de aptidão física relacionados à saúde em escolares ribeirinhos do Distrito de São Carlos, Município de Porto Velho, Rondônia. Dissertação (Mestrado em Ciências da Saúde) - Universidade de Brasília, Brasília, 2006.

HAYWOOD, K. M.; GETCHELL, N.. Desenvolvimento motor ao longo da vida. 6 ed. Artmed, 2010.

HENKES, C. M.; BORFE, L.; MURADÁS, R.; TORNQUIST, L.; BURGOS, M. S.. Aptidão física relacionada ao desempenho de escolares: estudo comparativo dos hemisférios norte - sul - leste - oeste da zona rural de Santa Cruz do Sul/RS. Cinergis, Santa Cruz do Sul, v.14, n.4, 2014.

IBGE. Instituto Brasileiro de Geografia e Estatística. Pesquisa de orçamentos familiares 2008-2009: antropometria e estado nutricional de crianças, adolescentes e adultos no Brasil. Rio de Janeiro: IBGE, 2010.

LERARIO, D. D. G.; GIMENO, S. G.; FRANCO, L. J.; IUNES, M.; FERREIRA, S. R. G.. Excesso de peso e gordura abdominal para a síndrome metabólica em nipo-brasileiros. Revista de Saúde Pública, v.36, n.1, p.4-11, 2002.
LUCIANO, A. P. BERTOLI, C. J.; ADAMI, F.; ABREU, L. C. Nível de Atividade Física em Adolescentes saudáveis. Revista Brasileira de Medicina do Esporte, v.22, n.3, p.191-194, 2016.

MALINA, R.; BOUCHARD, C.; BAR-OR, O.. Crescimento, maturação e atividade física. São Paulo: Phorte, 2009.

MASCARENHAS, L. P. G.; FERREIRA, A. B.; LIMA, V. A.; GRZELCZAK, M. T.. Estudo comparativo da aptidão física entre crianças de escola pública e particular: uma visão regional. Cinergis, Santa Cruz do Sul, v.14, n.3, 2014.

MONTORO, A. P. P. N.; LEITE, C. R.; ESPÍNDOLA, J. A.; ALEXANDRE, J. M.; REIS, M. S.; CAPISTRANO, R.; LISBOA, T.; BELTRAME, T. S.. Aptidão física relacionada à saúde de escolares com idade de 7 a 10 anos. Abcs Health Sciences, v.41, n.1, p.29-33, 2016. DOI: http://dx.doi.org/10.7322/abcshs.v41i1.842

NOGUEIRA, J. A. D.; PEREIRA, C. H.. Aptidão física relacionada à saúde de adolescentes participantes de programa esportivo. Rev. Bras. Educ. Fís. Esporte, São Paulo, v.28, n.1, p.31-40, 2014.

PELEGRINI, A.; SILVA, D. A. S.; PETROSKI, E. L.; GLANER, M. F.. Aptidão física relacionada à saúde de escolares brasileiros: dados do projeto esporte Brasil. Rev. Bras. Med. Esporte, São Paulo, v.17, n.2, p.92-96, 2011.

PEREIRA, T. A.; BERGMANN, G. G.. Aptidão Física Relacionada à Saúde e a Educação Física Escolar: Reflexões e experiências do PIBID UNIPAMPA. In: SALÃO INTERNACIONAL DE ENSINO, PESQUISA E EXTENSÃO, 4. Anais. 2013.

PETROSKI, E. L.; SILVA, A. F.; RODRIGUES, A. B.. Aptidão física relacionada a saúde em adolescentes brasileiros residentes em áreas de médio/baixo índice de desenvolvimento humano. Rev. Salud. Pública, v.13, n.2, p.219-228, 2011.

SANTOS, C. A.; CAMPOS, A. L. P.; SCHILD, J. F. G.. Comparação dos níveis de aptidão física e desempenho motor de estudantes de uma escola pública e uma escola privada da cidade de Pelotas. Revista Brasileira de Prescrição e Fisiologia do Exercício, São Paulo, v.7, n.41, p.440-448, 2013.

SANTOS, V. H. A.; NASCIMENTO, W. F.; LIBERALI, R.. O treinamento de resistencia muscular localizada com intervenção no emagrecimento. Revista Brasileira de Obesidade, Nutrição e Emagrecimento, São Paulo, v.2, n.7, p.34-43, 2008.

SILVA, R. J. S.. Capacidade física e testes motores relativos à promoção da saúde em crianças e adolescentes. Revista Brasileira de Cinantropometria e Desempenho Humano, v.5, n.1, p.75-84, 2003.

A CBPC - Companhia Brasileira de Produção Científica (CNPJ: 11.221.422/0001-03) detém os direitos materiais desta publicação. Os direitos referem-se à publicação do trabalho em qualquer parte do mundo, incluindo os direitos às renocões, expansões e disseminoc̃os da contribuiç̃o, bem como outros direitos subsidiários. Todos os trabalhos publicados eletronicamente poderão posteriormente ser publicados em coletâneas impressas sob coordenação da Sustenere Publishing da Companhia Brasileira de Produção Científica e seus parceiros autorizados. Os (as) autores (as) posteriormente ser publicados em coletâneas impressas sob coordenação da Sustenere Publishing, da Companhia Brasileira de Produção Cientifica e seus pa 